\title{
Adaptive impedance control with trajectory adaptation for minimizing interaction force
}

\author{
Jing Luo, Chenguang Yang^, Etienne Burdet, and Yanan Li
}

\begin{abstract}
In human-robot collaborative transportation and sawing tasks, the human operator physically interacts with the robot and directs the robot's movement by applying an interaction force. The robot needs to update its control strategy to adapt to the interaction with the human and to minimize the interaction force. To this end, we propose an integrated algorithm of robot trajectory adaptation and adaptive impedance control to minimize the interaction force in physical humanrobot interaction (pHRI) and to guarantee the performance of the collaboration tasks. We firstly utilize the information of the interaction force to regulate the robot's reference trajectory. Then, an adaptive impedance controller is developed to ensure automatic adaptation of the robot's impedance parameters. While one can reduce the interaction force by using either trajectory adaptation or adaptive impedance control, we investigate the task performance when combining both. Experimental results on a planar robotic platform verify the effectiveness of the proposed method.
\end{abstract}

\section{INTRODUCTION}

Advances in robotics and automation have promoted the development of research on human-robot collaboration [1] [2]. Physical human-robot interaction (pHRI) control has been wildly applied to assistive robots and it enables the human user to interact with the robot cooperatively, e.g. to manipulate a heavy object in a transportation task.

Impedance control or admittance control is a very useful control algorithm in the applications of pHRI. With impedance control, the stiffness, damping, and inertia parameters can be modified to regulate the robot's behaviours. In [3], a novel adaptive control approach was developed to detect the human's control intention and achieve the safe pHRI. For admittance control, the damping parameter was shown to be important in human-robot collaboration [4]. In [5], a variable admittance control with fuzzy inference was applied to a minimum jerk trajectory in a human-robot cooperation task. In addition, bio-signals have been widely used to recognize the human intention and to regulate the control strategy in the collaborative tasks. For example, an

Jing Luo is with the Key Laboratory of Autonomous Systems and Networked Control, College of Automation Science and Engineering, South China University of Technology, Guangzhou, 510640, China, and also with the Department of Bioengineering, Imperial College of Science Technology and Medicine, London SW7 2AZ, U.K.

Chenguang Yang is with Bristol Robotics Laboratory, University of the West of England, Bristol BS16 1QY, U.K. (e-mail: cyang@ieee.org).

Etienne Burdet is with the Department of Bioengineering, Imperial College of Science Technology and Medicine, London SW7 2AZ, U.K.

Yanan Li is with the Department of Engineering and Design, University of Sussex, Brighton BN1 9RH, U.K. electromyography-based control method was proposed to improve the interaction and coordination in the co-manipulative tasks [6]. Learning by demonstration (LbD) methods are also useful in pHRI and have been studied in the literature [7]. For example, an initial learning based on LbD method was proposed to update the robot trajectory and to achieve compliant control in the collaborative tasks [8].

To sum up, there is a basic idea to achieve efficient interaction by adapting the robot trajectory or regulating the control parameters such as stiffness and damping in impedance control. In line with this idea, we explore the possibility to achieve efficient interaction by combining trajectory adaptation and control parameters regulation.

As shown in Fig. 1, the context of this paper is also related to shared control which investigates how to allocate control authority for the robot and human in pHRI [9] [10] [11]. For example, in [9], a shared control method using reinforcement learning was proposed to adjust the control weight of the human user dynamically. An adaptive shared control method was developed to improve the user's self-motivated ambulatory ability for a robotic walker in rehabilitation training [10]. However, these methods have not considered the user performance criterion and the interaction profile. Therefore, we present a new control framework by considering the user performance criterion. Validation of the proposed method is performed on a robotic platform to be introduced in the experiment section.

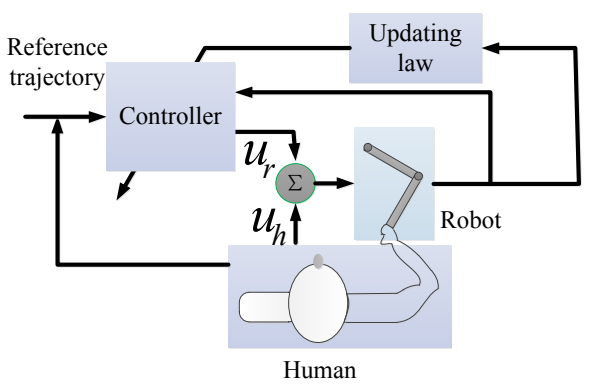

Fig. 1. Allocation of control authority in physical human-robot interaction.

The rest of this paper is organized as below. Section II describes the problem formulation and the proposed trajectory adaptation and impedance control method. Section III presents the validation results. Section IV summarizes the conclusion and discussions. 


\section{PROBLEM FORMULATION AND PROPOSED METHOD}

\section{A. System description}

In this paper, we consider a scenario where the robot is expected to follow the human motion and to minimize the interaction force, e.g. in a transportation task in Fig. 2. The objective is to design a control strategy for the robot to adapt to the interaction profile between the robot and the human user in the process of pHRI.

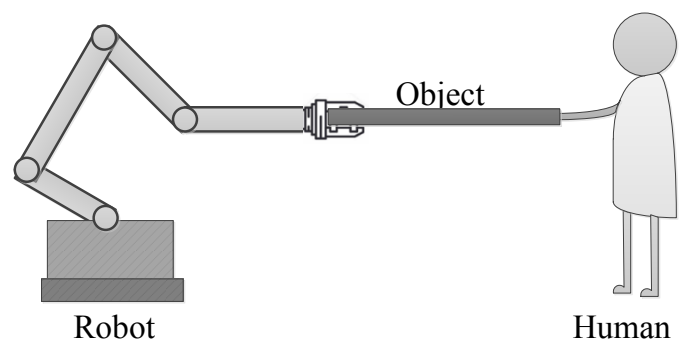

Fig. 2. A scenario of human-robot transportation task.

Considering the human force as an input of the system, the system dynamics can be written as

$$
M(x) \ddot{x}+C(x, \dot{x}) \dot{x}+G(x)=u+F_{h}
$$

where $x$ denotes the robot position, $u$ is the robot's control input, $F_{h}$ is the interaction force, and $M, C, G$ are the system inertia, Coriolis and centrifugal, and gravity matrices.

The structure of the proposed method is shown in Fig. 3. It can be seen that the robot trajectory can be adjusted according to the interaction force. The impedance controller can be updated based on the trajectory tracking performance to allocate the control authority for the robot automatically. This novel method is proposed to achieve the aim of reducing the interaction force according to the interaction profile. In the following two subsections, the detailed trajectory adaptation and impedance control method will be presented.

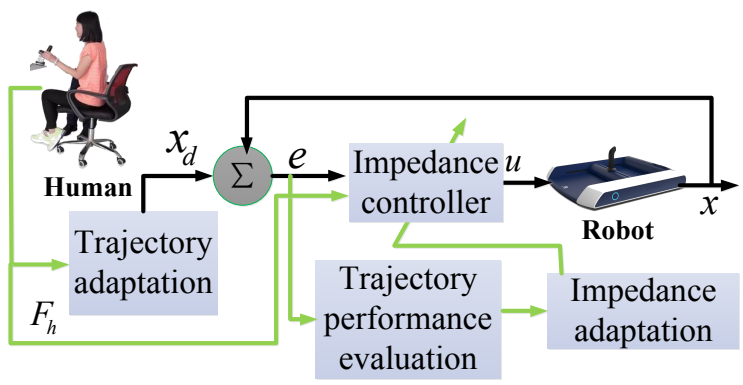

Fig. 3. Overall structure of the proposed algorithm.

\section{B. Trajectory adaptation}

Since the dynamics of the robotic system is affected by the HRI profile, it is essential to update the reference trajectory of the robot according to its current state and the interaction force. In particular, a function can be assumed as follows:

$$
x_{d}=\phi\left(x, F_{h}\right)
$$

where $x_{d}$ is the robot's reference trajectory and $\phi$ is a function to be designed.

In this paper, an explicit law is designed to update the trajectory of the robot by using the interaction force information. The updating law for the robot's trajectory can be defined as below:

$$
x_{d}=x+\beta_{1} F_{h}
$$

where $\beta_{1}=M_{1} \alpha_{1}$ represents an open parameter to regulate the interaction force. $M_{1}$ denotes a constant for adjusting $\alpha_{1}$, while the detailed process to design $\alpha_{1}$ is presented as below.

Since the interaction force is related to the position error, we construct a function to represent the relationship between the interaction force and position error. Inspired by [12], it can be defined as

$$
\xi_{1}=K_{1} e^{2}
$$

where $K_{1}$ is a parameter to regulate the position error $e$ and $\xi_{1}$ is a performance evaluation index.

Then, an index function $J\left(\xi_{1}\right)$ can be obtained as

$$
J\left(\xi_{1}\right)=\sum_{j=1}^{i} \xi_{1}(j) e^{-\mu_{1}(i-j) \Delta t}
$$

where $\mu_{1}$ denotes a parameter to regulate the forgetting rate, $\Delta t$ is a sampling time of the system, and $i$ is the number of sampling times.

With the above definitions, the parameter $\alpha$ can be computed through the following equation:

$$
\alpha_{1}(i)=1-e^{-\nu_{1} J\left(\xi_{1}\right)}
$$

where $\nu_{1}$ is used to adjust the adaptation of the interaction force with $\mu_{1}$.

It is noted that the robot can automatically update the reference trajectory according to the change of the interaction force. As to be shown in the experiment section, this adaptation law can achieve a smooth interaction between the human and the robot.

\section{Adaptive impedance control}

For impedance control, we design a similar variable $\alpha_{2}$ to update the impedance parameter.

In particular, the model of impedance control can be represented as

$$
F_{i m}=K_{c} e+K_{d} \dot{e}
$$

with

$$
K_{c}=K_{c 0}+\Delta K
$$

where $F_{i m}$ denotes the input of the impedance control and it is noted that $u=F_{i m}$ as in (1). $K_{c}$ is a variable stiffness. $K_{c 0}$ is a nominal stiffness, which needs to be designed according to the experimental results. $K_{d}$ represents a damping that is fixed as a constant in this paper.

$\Delta K$ is designed as below:

$$
\Delta K=\beta_{2}\left|F_{h}\right|
$$


where $\beta_{2}=M_{2} \alpha_{2}$ denotes a parameter to regulate the stiffness of the impedance controller. $M_{2}$ is a constant used for adjusting $\alpha_{2}$.

Similarly as for trajectory adaptation, the process of computation of $\alpha_{2}$ is presented in the following. First, we define a performance evaluation index $\xi_{2}$ as below:

$$
\xi_{2}=K_{2} e^{2}
$$

where $K_{2}$ is a parameter to adjust the weight of the tracking error.

With a given sampling time, we can calculate the performance index $J\left(\xi_{2}\right)$ as

$$
J\left(\xi_{2}\right)=\sum_{j=1}^{i} \xi_{2}(j) e^{-\mu_{2}(i-j) \Delta t}
$$

where $\mu_{2}$ denotes a parameter to regulate the forgetting rate.

Then, the parameter $\alpha_{2}$ can be calculated according to the following equation:

$$
\alpha_{2}(i)=e^{-\nu_{2} J\left(\xi_{2}\right)}
$$

where $\nu_{2}$ is used to adjust the adaptation of the interaction force.

It is noted that $\alpha_{2}$ is utilized to adjust the user's interaction profile. The adjustment of impedance parameter based on the performance evaluation is automatic in the control loop. Based on the above description, it can be seen that the impedance control input accounts the human input (interaction force) and user performance evaluation index.

With trajectory adaptation and adaptive impedance control, the proposed framework can address the requirement of decreasing the interaction force and enables to the robot to "actively" follow the human movement. This statement will be justified by the experimental results in the following section.

\section{EXPERIMENTS}

In this section, we validate the performance of the proposed method in different experiments.

\section{A. Experimental setup}

In the experiments, we emulate the transportation task through pHRI (Fig. 2). The aim of this transportation task for the robot is to minimize the interaction force for achieving a natural interaction with its human user. In specific, when the human user changes their control intention, the robot needs to update its reference trajectory and control strategy to ensure the success of this task. In order to simplify the experimental setup, we assume that the object is a point mass, so the human user can perform this task by holding the endeffector of the robot. Fig. 4 shows the experimental platform, where H-MAN (ARTICARES Pte Ltd in Singapore) is used to interact with the human user. H-MAN is a planar robot containing a body, a slide and a handle. An ATI Mini 40 force sensor is installed on the end of the handle to measure the interaction force. H-MAN communicates with a computer through Transmission Control Protocol (TCP). The force sensor sends the data to the computer by using a conversion equipment (ATI Net Box) through TCP. All devices are performed on the Windows 7 operation system with Visual Studio 2013 software platform.

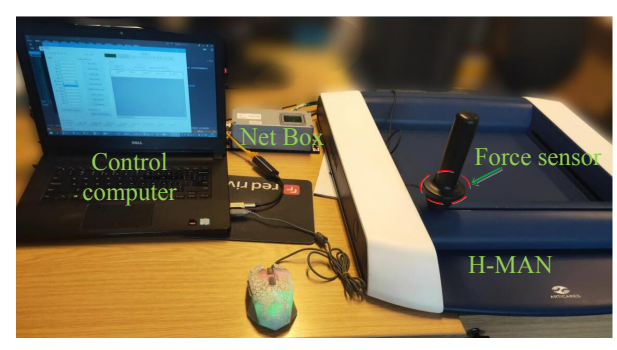

Fig. 4. Experimental platform.

\section{B. Experimental results}

Three different experiments are performed with different parameters to demonstrate the effectiveness of trajectory adaptation and impedance control.

1) Experiment 1: trajectory adaptation only: In this experiment, the robot reference trajectory is adapted with constant impedance parameters, i.e. $K_{c}=200, K_{d}=50$. In order to evaluate the influence of parameter $\beta_{1}$, we consider three cases:

- Case 1: $\beta_{1}=0$.

- Case 2: constant $\beta_{1}=0.1$.

- Case 3: variable $\beta_{1}=0.1 \alpha_{1}$.

Other parameters are set as $K_{1}=10, K_{2}=K_{3}=$ $K_{4}=0.05$, and $\nu_{1}=\mu_{1}=\nu_{2}=\mu_{2}=0.05$. Figs. 5-7 show the performance of robot trajectory adaptation under the condition of different parameters $\beta_{1}$. In Case 1 , the desired trajectory is a sinusoid, shown in Fig. 5(a). The human user can follow the desired trajectory or change the robot's motion based on their control intention. It can be seen that the interaction force and tracking error are very large in Figs. 5(b)-5(c), showing that the human user finds difficult to cooperate with the robot.

From the results in Cases 2-3, we can see that the interaction force starts with a big value during the interaction. As the task continues, the interaction force decreases dramatically and indicates a periodic behaviour, showing that the cooperation between the human and the robot becomes easier in these cases.

In Experiment 1, we can see that the average absolute value of the interaction force is the smallest in Case 2 (Table I), while the tracking errors for all three cases are relatively large.

Based on these results, it can be concluded that the human can interact with the robot with a small interaction force by adapting the robot trajectory. However, there exists a relatively large tracking error when using the trajectory adaptation method only.

2) Experiment 2: adaptive impedance control only: In Experiment 2, the desired trajectory is set as $x_{d}=1-$ $\cos (2 \pi t / 3)$ and the stiffness parameter is $K_{c}=200+\beta_{2}\left|F_{h}\right|$. In order to evaluate the influence of parameter $\beta_{2}$, we consider the following three cases:

- Case 4: $\beta_{2}=0$. 


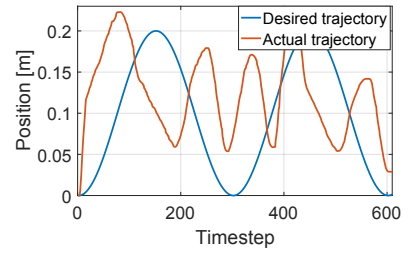

(a) Robot Trajectory. (b) Trajectory error.
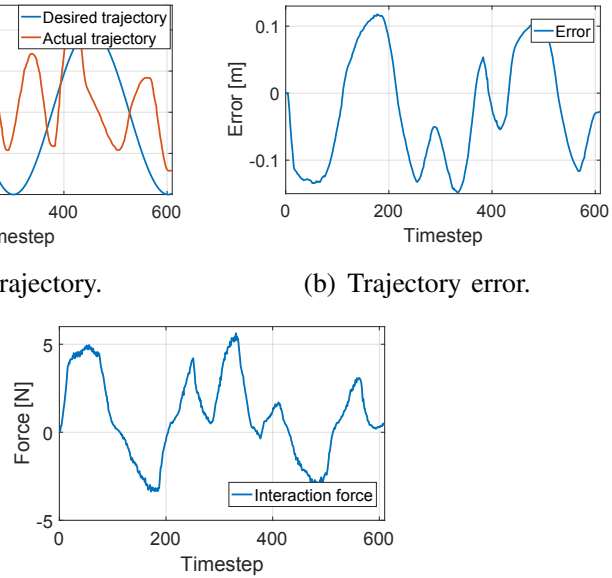

(c) Interaction force.

Fig. 5. Experiment 1: the experimental results with $\beta_{1}=0$ in Case 1.

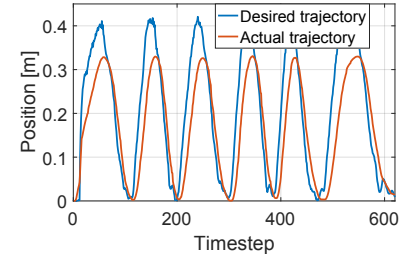

(a) Robot Trajectory.

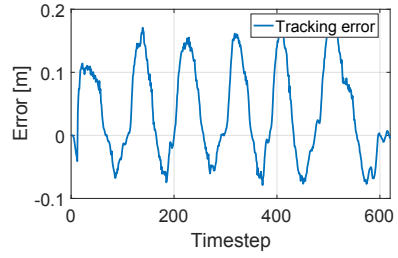

(b) Trajectory error.

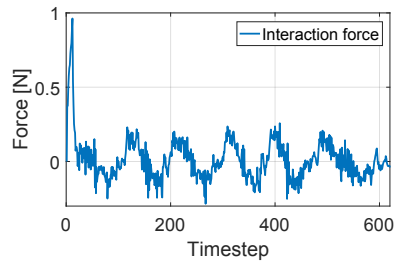

(c) Interaction force.

Fig. 6. Experiment 1: the experimental results with $\beta_{1}=0.1$ in Case 2 .

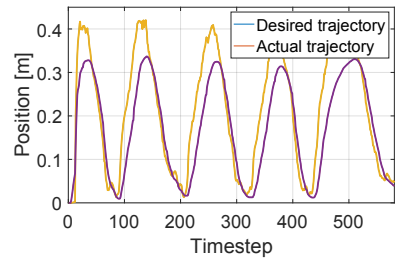

(a) Robot Trajectory.

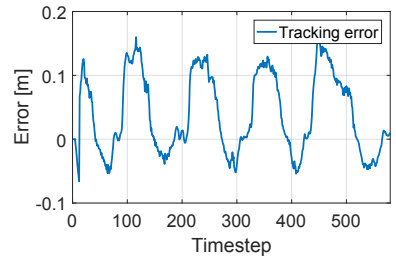

(b) Trajectory error.

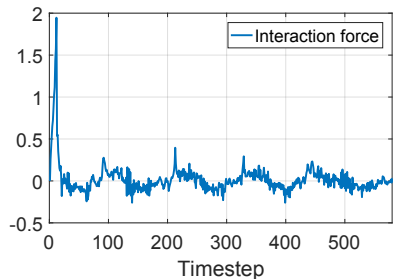

(c) Interaction force.

Fig. 7. Experiment 1: the experimental results with $\beta_{1}=0.1 \alpha_{1}$ in Case 3 .
- Case 5: constant $\beta_{2}=50$.

- Case 6: variable $\beta_{2}=50 \alpha_{2}$.

Other parameters are set the same as in Experiment 1. From Figs. 8-10 respectively for Cases 4-6 and Table I, we can see that all the interaction forces are relatively large when the human user follows the desired trajectory of the robot and the tracking errors are relatively small, although they are smaller than the counterparts in Experiment 1. It can be interpreted that when the human user changes their control intention, they need to apply a large force to achieve this aim. As the reference trajectory does not update, the tracking error also becomes large. In this sense, it is difficult to achieve a small interaction force by only using adaptive impedance control.

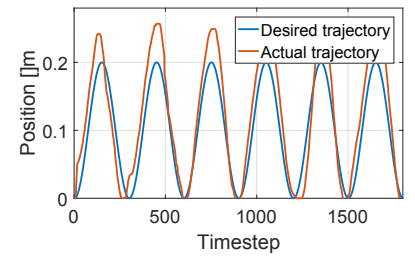

(a) Robot Trajectory.

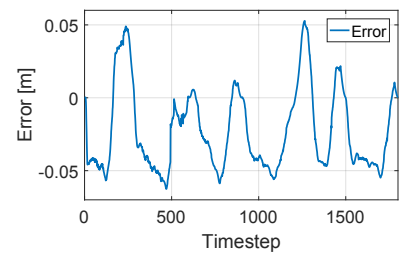

(b) Trajectory error.

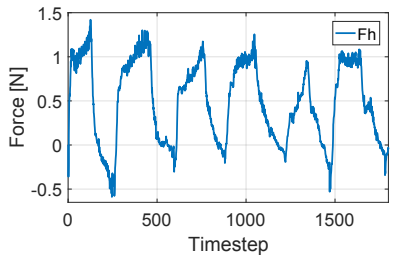

(c) Interaction force.

Fig. 8. Experiment 2: the experimental results with $\beta_{2}=0$ in Case 4 .

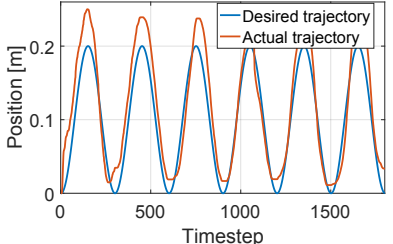

(a) Robot Trajectory.

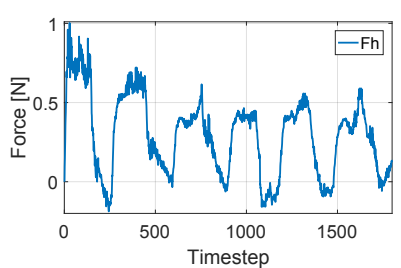

(c) Interaction force.

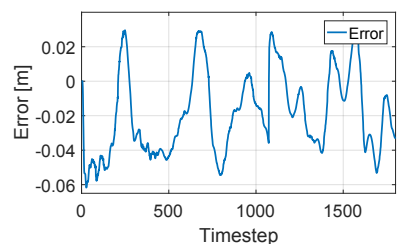

(b) Trajectory error.

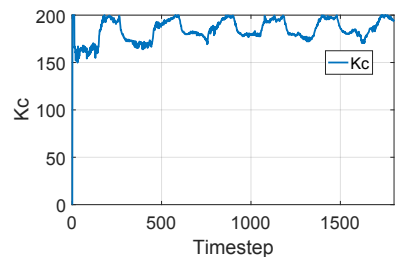

(d) Parameter of impedance.
Fig. 9. Experiment 2: the experimental results with $\beta_{2}=50$ in Case 5 .

3) Experiment 3: trajectory adaptation and adaptive impedance control: This experiment is performed under the condition of robot trajectory adaptation $x_{d}=x+\beta_{1} F_{h}$ and adaptive impedance control $K_{c}=K_{c 0}+\Delta K=K_{c 0}+$ 


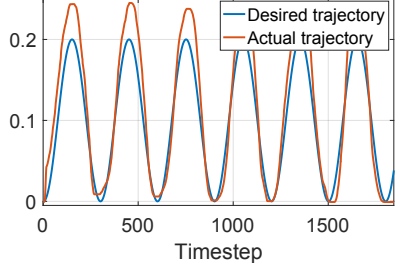

(a) Robot Trajectory.

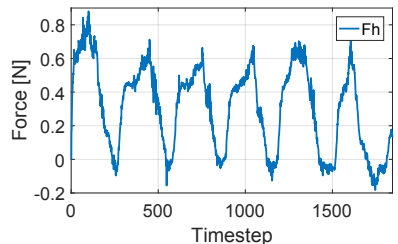

(c) Interaction force

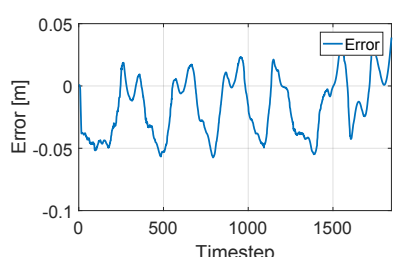

(b) Trajectory error.

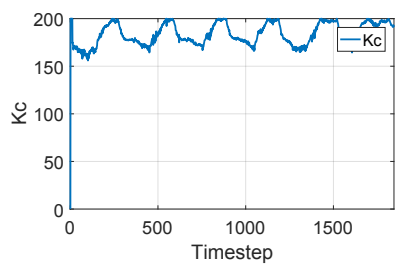

(d) Parameter of trajectory adaptation.
Fig. 10. Experiment 2: the experimental results with $\beta_{2}=50 \alpha 2$ in Case 6 .

$\beta_{2}\left|F_{h}\right|$, as elaborated in the approach section. Specifically, the following four cases are considered:

- Case 7: constant $\beta_{1}=0.1$ and constant $\beta_{2}=50$.

- Case 8: constant $\beta_{1}=0.1$ and variable $\beta_{2}=50 \alpha_{2}$.

- Case 9: variable $\beta_{1}=0.1 \alpha_{1}$ and constant $\beta_{2}=50$.

- Case 10: variable $\beta_{1}=0.1 \alpha_{1}$ and variable $\beta_{2}=50 \alpha_{2}$.

Other experimental parameters are set the same as in Experiment 1. Figs. 11-13 show the experimental results in the cases of trajectory adaptation and variable parameters $\beta_{1}$ or $\beta_{2}$. It can be seen that the interaction force becomes small, and the position error also becomes small and consistent. Similarly, the profile of $K_{c}$ has the same trend as the change of the interaction force. It can be concluded that the human can change their control intention with a small interaction force while the robot achieves a small tracking error, as the robot's reference trajectory and impedance parameters can be updated according to the change of the interaction profile.

In Fig. 14, we can see the results in the case of trajectory adaptation $\beta_{1}=0.1 \alpha_{1}$ and constant $\beta_{2}=50 \alpha_{2}$. While similar results are achieved as that in Cases 7-9, the tracking error and interaction force are smallest in the four cases. Additionally, by calculating the average absolute value of tracking error and interaction force, we find that the best performance is achieved in Case 10 (Table I). Based on these results, it can concluded that the robot can collaborate with the human user best in the case of variable $\beta_{1}$ and $\beta_{2}$.

\section{Conclusions}

In this paper, we propose a new approach based on robot trajectory adaptation and adaptive impedance control for the robot to reduce the interaction force, potentially useful for human-robot collaboration such as in a transportation task. Results in experiments 1-2 show that both trajectory adaptation and adaptive impedance control can be used to reduce the interaction force individually, and experiments demonstrate

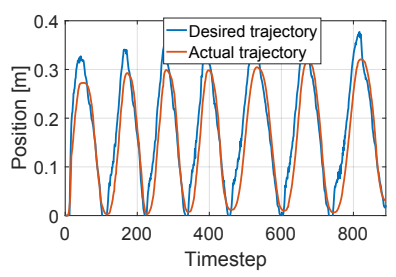

(a) Robot Trajectory.

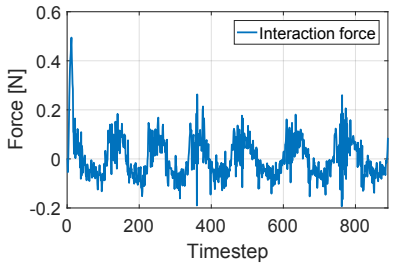

(c) Interaction force.

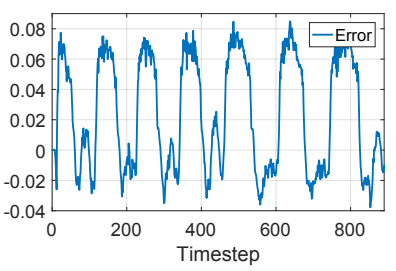

(b) Trajectory error.

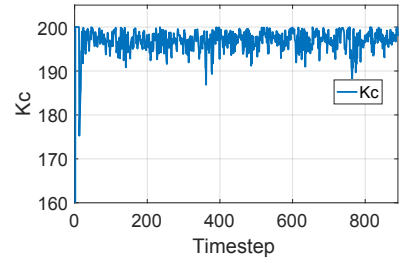

(d) Parameter of trajectory adaptation.
Fig. 11. Experiment 3: the experimental results with $\beta_{1}=0.1$ and $\beta_{2}=50$ in Case 7.

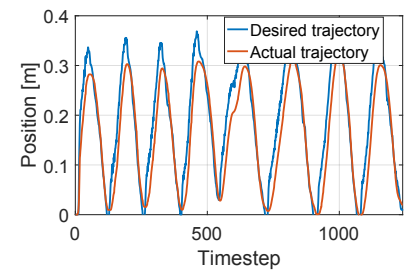

(a) Robot Trajectory.

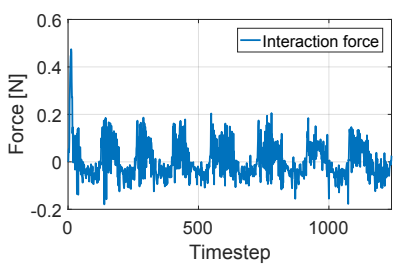

(c) Interaction force

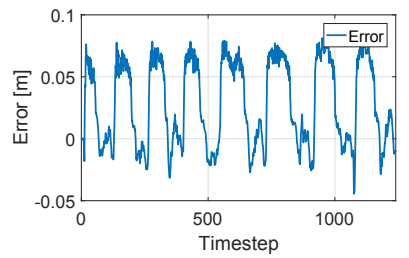

(b) Trajectory error.

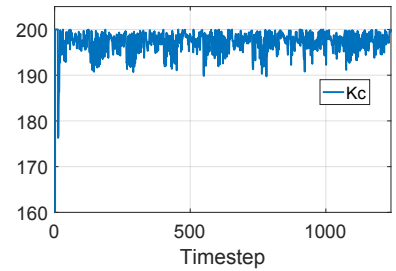

(d) Parameter of trajectory adaptation.
Fig. 12. Experiment 3: the experimental results with $\beta_{1}=0.1$ and $\beta_{2}=$ $50 \alpha_{2}$ in Case 8.

that it is reasonable to combine these two methods to further minimize not only the interaction force, but also the tracking error.

Compared with the existing methods, the developed method does not only consider the tracking error information, interaction profile, but also the performance evaluation index. As shown by the experimental results, when the human physically interacts with the robot in a cooperative task, the proposed framework can ensure a small interaction force and achieve a smooth interaction. In comparison, it is difficult to achieve a small interaction force with a small tracking error in pHRI with either trajectory adaptation only or adaptive impedance control only.

It is noted that the robustness of the proposed framework needs to be analysed in detail in the future. In addition, the learning method will be introduced to update the impedance 
TABLE I

AVERAGE ABSOLUTE VALUE FOR POSITION ERROR AND INTERACTION FORCE IN EXPERIMENTS 1, 2 AND 3.

\begin{tabular}{|c|c|c|c|c|c|c|c|c|c|c|}
\hline Parameters description & $\begin{array}{c}\text { Case } \\
1\end{array}$ & $\begin{array}{c}\text { Case } \\
2\end{array}$ & $\begin{array}{c}\text { Case } \\
3\end{array}$ & $\begin{array}{c}\text { Case } \\
4\end{array}$ & $\begin{array}{c}\text { Case } \\
5\end{array}$ & $\begin{array}{c}\text { Case } \\
6\end{array}$ & $\begin{array}{c}\text { Case } \\
7\end{array}$ & $\begin{array}{c}\text { Case } \\
8\end{array}$ & $\begin{array}{c}\text { Case } \\
9\end{array}$ & $\begin{array}{l}\text { Case } \\
10\end{array}$ \\
\hline $\begin{array}{l}\text { Average absolute value } \\
\text { of position error [m] }\end{array}$ & 0.0793 & 0.0674 & 0.0571 & 0.0317 & 0.0263 & 0.0239 & 0.0363 & 0.0352 & 0.0335 & 0.0331 \\
\hline $\begin{array}{l}\text { Average absolute value } \\
\text { of interaction force }[\mathrm{N}]\end{array}$ & 2.0396 & 0.0989 & 0.0926 & 0.5461 & 0.3154 & 0.3274 & 0.0660 & 0.0588 & 0.0674 & 0.0485 \\
\hline
\end{tabular}

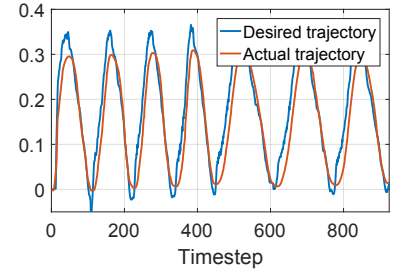

(a) Robot Trajectory.

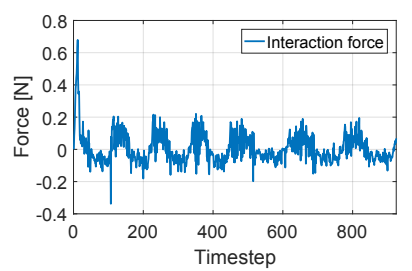

(c) Interaction force.

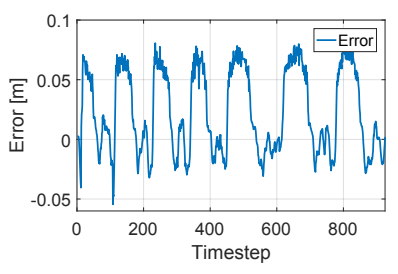

(b) Trajectory error.

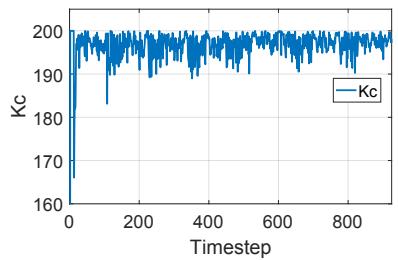

(d) Parameter of trajectory adaptation.

Fig. 13. Experiment 3: the experimental results with $\beta_{1}=0.1 \alpha 1$ and $\beta_{2}=$ 50 in Case 9.

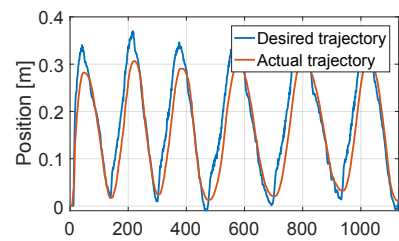

(a) Robot Trajectory.

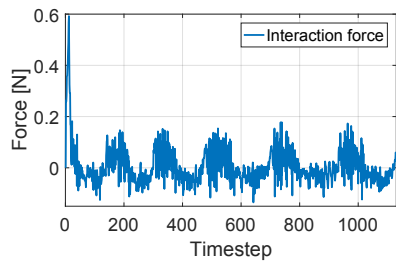

(c) Interaction force.

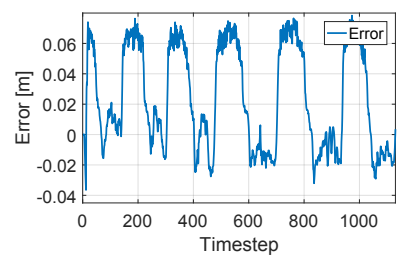

(b) Trajectory error.

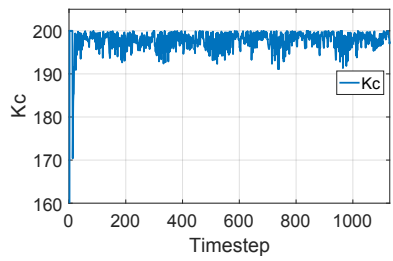

(d) Parameter of trajectory adaptation.
Fig. 14. Experiment 3: the experimental results with $\beta_{1}=0.1 \alpha 1$ and $\beta_{2}=$ $50 \alpha_{2}$ in Case 10.

control and to learn the human's interaction behaviours in a higher level of decision marking [13] [14].

\section{REFERENCES}

[1] J. Luo, Z. Lin, Y. Li, and C. Yang, "A teleoperation framework for mobile robots based on shared control," IEEE Robotics and Automation Letters, vol. 5, no. 2, pp. 377-384, 2019.
[2] J. Luo, C. Yang, N. Wang, and M. Wang, "Enhanced teleoperation performance using hybrid control and virtual fixture," International Journal of Systems Science, vol. 50, no. 3, pp. 451-462, 2019.

[3] X. Li, Y. Pan, G. Chen, and H. Yu, "Adaptive human-robot interaction control for robots driven by series elastic actuators," IEEE Transactions on Robotics, vol. 33, no. 1, pp. 169-182, 2016.

[4] R. Ikeura, H. Monden, and H. Inooka, "Cooperative motion control of a robot and a human," in Proceedings of 1994 3rd IEEE International Workshop on Robot and Human Communication, pp. 112-117, IEEE, 1994.

[5] F. Dimeas and N. Aspragathos, "Fuzzy learning variable admittance control for human-robot cooperation," in 2014 IEEE/RSJ International Conference on Intelligent Robots and Systems, pp. 4770-4775, IEEE, 2014.

[6] L. Peternel, N. Tsagarakis, and A. Ajoudani, "A human-robot comanipulation approach based on human sensorimotor information," IEEE Transactions on Neural Systems and Rehabilitation Engineering, vol. 25, no. 7, pp. 811-822, 2017.

[7] L. Rozo, S. Calinon, D. G. Caldwell, P. Jimenez, and C. Torras, "Learning physical collaborative robot behaviors from human demonstrations," IEEE Transactions on Robotics, vol. 32, no. 3, pp. 513-527, 2016.

[8] B. Nemec, N. Likar, A. Gams, and A. Ude, "Human robot cooperation with compliance adaptation along the motion trajectory," Autonomous robots, vol. 42, no. 5, pp. 1023-1035, 2018.

[9] W. Xu, J. Huang, Y. Wang, C. Tao, and X. Gao, "Research of reinforcement learning based share control of walking-aid robot," in Proceedings of the 32nd Chinese Control Conference, pp. 5883-5888, IEEE, 2013.

[10] J. Ye, G. Chen, Q. Liu, L. Duan, W. Shang, X. Yao, J. Long, Y. Wang, Z. Wu, and C. Wang, "An adaptive shared control of a novel robotic walker for gait rehabilitation of stroke patients," in 2018 IEEE International Conference on Intelligence and Safety for Robotics (ISR), pp. 373-378, IEEE, 2018.

[11] H. Yu and S. Dubowsky, "Omnidirectional vehicle with offset wheel pairs," Apr. 1 2003. US Patent 6,540,039.

[12] H. Yu, M. Spenko, and S. Dubowsky, "An adaptive shared control system for an intelligent mobility aid for the elderly," Autonomous Robots, vol. 15, no. 1, pp. 53-66, 2003.

[13] M. Khoramshahi and A. Billard, "A dynamical system approach to task-adaptation in physical human-robot interaction," Autonomous Robots, vol. 43, no. 4, pp. 927-946, 2019.

[14] E. Gribovskaya, A. Kheddar, and A. Billard, "Motion learning and adaptive impedance for robot control during physical interaction with humans," in 2011 IEEE International Conference on Robotics and Automation, pp. 4326-4332, IEEE, 2011. 